\title{
EDITORIAL
}

\section{Measuring Successful Treatment of Irritable Bowel Syndrome: Is "Satisfactory Relief" Enough?}

\author{
Philip Schoenfeld, M.D., MSEd., M.Sc. (Epi) ${ }^{1}$ and Nicholas J. Talley, M.D., Ph.D. ${ }^{2}$ \\ ${ }^{1}$ Division of Gastroenterology, University of Michigan School of Medicine, Ann Arbor, Michigan, and ${ }^{2}$ Division \\ of Gastroenterology, Mayo Clinic College of Medicine
}

\begin{abstract}
The treatment options for the irritable bowel syndrome (IBS) are expanding as new therapies, including probiotics and serotonin receptor agents, become available. Before any new agents gain widespread use, they must be studied in appropriately designed clinical trials. Symptom improvement remains the key clinically but the best technique to measure symptom improvement is unclear. Many IBS therapy studies have used a binary endpoint such as "Have you had satisfactory relief of your IBS symptoms in the past week? Yes/No?" The study by Whitehead and colleagues in this issue suggests that "satisfactory relief" is affected by baseline symptom severity and may not always truly reflect the symptom burden. Future research needs to determine whether "satisfactory relief" is truly adequate, or whether alternatives such as the proportion of patients achieving a $\geq 50 \%$ reduction in symptom severity would represent a superior approach to capture clinically important improvement.
\end{abstract}

(Am J Gastroenterol 2006;101:1066-1068)

Based on recent evidence, it appears likely that irritable bowel syndrome (IBS) is an organic gut-brain disorder; key pathophysiologic defects in subsets of IBS patients include increased sensitivity to colonic distention and altered central pain processing, abnormal colonic serotonin receptor signaling, low grade colonic inflammation, and possibly an abnormal enteric bacterial-immune interface (1-4). However, there is as yet no accepted biological disease marker. Furthermore, there is no obvious marker to utilize when assessing the severity of IBS symptoms or IBS symptom improvement, unlike gastroesophageal reflux disease (GERD) where the severity of esophagitis can be assessed endoscopically and healing documented. The functional bowel disease severity scale purports to assess IBS severity but was validated only against physician judgment and is confounded by office visits over the previous 6 months, which limits its value (5). Therefore, IBS researchers and clinicians must still rely upon symptom improvement to measure successful treatment of IBS.

Given this dilemma, the Rome Committee on treatment trials (6) recommended that the primary endpoint in IBS therapy trials was best assessed by a global subjective improvement in IBS symptoms. This endpoint had the advantage of potentially encompassing improvement in the multiple symptoms of IBS, including abdominal discomfort, bloating, and stool consistency. The U.S. Food and Drug Administration has accepted subjective global improvement in IBS symptoms as a primary endpoint (7), especially when assessed using a binary (yes/no) measure such as "In the last seven days, have you had adequate (or satisfactory) relief of your IBS symptoms?" We must ask if this simple binary endpoint, "satisfactory relief of IBS symptoms," is optimal to determine if an IBS patient has been successfully treated.
In this issue of the journal, Whitehead and colleagues (8) report their analysis of patients receiving standard medical care for IBS at a large health maintenance organization (9). In this secondary analysis, the authors looked at the results of a "satisfactory relief" endpoint measured with a binary question. They compared these results to a seven-point Likert scale rating subjective improvement in gastrointestinal symptoms and to the magnitude of symptom reduction using a valid IBS symptom severity questionnaire. The investigators sought to determine if initial IBS symptom severity influences the likelihood of achieving "satisfactory relief" and if it influenced the magnitude of IBS symptom reduction. The investigators found that patients with mild IBS symptoms at baseline were much more likely to achieve "satisfactory relief" of IBS symptoms after treatment compared to patients with severe IBS symptoms at baseline ( $72 \%$ vs $44 \%$, respectively; $p<0.001)$. However, when satisfactory response to therapy was determined by a $\geq 50 \%$ reduction in IBS symptoms from baseline, patients with mild IBS symptoms and patients with severe IBS symptoms [defined by an IBS symptom severity scale (10)] had a similar likelihood of achieving this endpoint ( $26 \%$ vs $23 \%$, respectively; $p=n s$ ). Notably, by adjusting the IBS symptom score for baseline values, the deck was stacked against satisfactory relief here, but this is the point: satisfactory relief was confounded by baseline symptom severity in this study. Therefore, if achievement of "satisfactory relief" is the primary endpoint of an IBS therapy trial, then it may be relatively easy to achieve this endpoint if most of the patients have "mild" IBS symptoms. However, a $50 \%$ reduction in symptoms in a patient with severe IBS symptoms is potentially an important finding and probably represents a clinically meaningful change even if the patient 
with severe IBS symptoms does not achieve "satisfactory relief." Given these results, Whitehead and colleagues speculate that binary endpoints measuring "satisfactory relief" may not be optimal to detect a clinically meaningful change and hence may miss real benefits in clinical trials. However, the limitations of this study (8) must be remembered. This was an observational study rather than a randomized controlled trial and may be subject to numerous biases that could have exaggerated the results.

If "satisfactory relief" endpoints are confounded by initial IBS symptom severity, then what should be the primary endpoint? A 50\% reduction in global symptom severity? Complete or near complete resolution of symptoms? Significant improvement in the score of a validated quality-oflife questionnaire? The recommendations of the Rome Committee on treatment trials (6) provide some guidance about this topic.

1. A subjective global assessment of IBS symptom improvement should be used as the primary endpoint. This subjective global endpoint should allow the patient to assess improvement in multiple IBS symptoms (e.g., abdominal discomfort, bloating, stool consistency, stool frequency, etc.) because improvement in a single symptom (e.g., stool frequency) may not be adequate to demonstrate true improvement in IBS.

2. IBS therapy trials should measure individual IBS symptoms (e.g., abdominal discomfort, bloating, etc.) as secondary endpoints. If patients demonstrate significant improvement in the primary endpoint of subjective global improvement in IBS symptoms, then this finding is reinforced if significant improvement is also demonstrated in individual IBS symptoms.

3. IBS therapy trials should also measure symptom improvement with a validated quality-of-life questionnaire. If a treatment is associated with a significant improvement in quality of life, then this also reinforces the benefits of an IBS therapy.

Various quality-of-life questionnaires have been applied in IBS therapy trials. Some questionnaires are generic (e.g., SF36) and can be utilized to measure changes in quality of life for any disorder, such as depression or diabetes mellitus. However, these questionnaires evaluate such broad categories that they may not be able to measure clinically important changes in quality of life for a specific disorder like IBS. IBS-specific questionnaires, such as the IBS-QOL (11) and the IBS severity scoring system (IBS-SSS) (10), are available for use in IBS patients, but we do not know what constitutes a "clinically important improvement" in scores on these validated questionnaires. Complete symptom relief has proven a very useful endpoint in GERD trials assessing proton pump inhibitor therapy. Certainly, complete relief of symptoms is convincing evidence of efficacy if placebo is beaten; further, we strongly recommend results on complete relief of active therapy and placebo should always be reported in clinical trials of IBS treatments as a secondary endpoint. However, it seems unlikely that current agents would frequently completely resolve all IBS symptoms even if the treatment produced "satisfactory relief." Therefore, complete relief of symptoms is not optimal as a primary endpoint in IBS therapy trials now.

The value of "satisfactory relief" in IBS therapy trials relates to its ability to integrate various symptoms and the impact of therapy on various symptoms; it is also easy to understand and it fits with the practice of medicine in the office setting. When we see patients for return visits in our office, our first question is frequently a global one such as "How are you doing?" If a patient with IBS replies that they are doing much better in terms of their IBS symptoms, then this question seems to usefully provide a rapid assessment of relevant symptom relief. A positive answer suggests that the patient has undergone a clinically important improvement in their symptoms. Therefore, we believe that a binary assessment of "satisfactory relief" endpoints is useful in IBS therapy trials. Based on the data provided by Whitehead and colleagues (5), we suggest it is important for investigators to also measure IBS symptom severity utilizing a validated scale. Investigators should consider reporting the proportion of IBS patients with mild, moderate, or severe symptoms, since this may influence the proportion of patients who undergo "satisfactory relief" of their IBS symptoms. Furthermore, we agree with Whitehead and colleagues (5) that measurement of $\geq 50 \%$ reduction in IBS symptoms is most likely associated with a clinically important improvement in IBS symptoms and that this is worth assessing as a secondary endpoint; more data are needed before its use as a primary endpoint could be considered. Consistent with the Rome Committee recommendations (3), we also agree that individual IBS symptoms of abdominal discomfort, bloating, and stool consistency, should be measured as secondary endpoints, and this can usually be performed satisfactorily with Likert scales or visual analog scales. However, we do not think that these should be primary endpoints because minimally clinically important improvements in individual symptoms measured by visual analog scales or Likert scales have not been defined in IBS. Finally, the use of validated quality-of-life questionnaires should be considered a tertiary endpoint at this time since the minimal clinically important improvement in IBS-specific quality-oflife scores also remain unclear.

Reprint requests and correspondence: Philip Schoenfeld, VAMC 111-D, 2215 Fuller Rd., Ann Arbor, MI 48105.

Received November 15, 2005; accepted December 7, 2005.

\section{REFERENCES}

1. O’Mahony L, McCarthy J, Kelly P, et al. Lactobacillus and bifidobacterium in irritable bowel syndrome: Symptom responses and relationship to cytokine profiles. Gastroenterology 2005;128:541-51.

2. Caldarella MP, Milano A, Laterza F, et al. Visceral sensitivity and symptoms in patients with constipation- or 
diarrhea-predominant irritable bowel syndrome (IBS): Effect of a low-fat intraduodenal infusion. Am J Gastroenterol 2005;100(2):383-9.

3. Coates MD, Mahoney CR, Linden DR, et al. Molecular defects in mucosal serotonin content and decreased serotonin reuptake transporter in ulcerative colitis and irritable bowel syndrome. Gastroenterology 2004;126:165764.

4. Malinen E, Rintiila T, Kanjander K, et al. Analysis of the fecal microbiota of irritable bowel syndrome patient and healthy controls with real-time PCR. Am J Gastroenterol 2005;100(2):373-82.

5. Drossman DA, Zhiming LI, Toner BB, et al. Functional bowel disorders: A multicenter comparison of health status and development of illness severity index. Dig Dis Sci 1995;40:986-95.

6. Veldhuyzen van Zenten SJO, Talley NJ, Bytzer P, et al. Design of treatment trials for the functional gastrointestinal disorders. In: Drossman DA, Corazziari E, Talley NJ, et al. eds. Rome II. functional gastrointestinal disorders: Diagnosis, pathophysiology, and treatment - a multinational consensus. McLean, VA: Degnon Associates; 2000:577622.

7. Mangel AW, Hahn VA, Heath AT, et al. Adequate relief as an endpoint in clinical trials of irritable bowel syndrome. J Int Med Res 1998;26:76-81.

8. Whitehead WE, Palsson OS, Levy RL, et al. Reports of "satisfactory relief" by IBS patients receiving usual medical care are confounded by baseline symptom severity and do not accurately reflect symptom improvement. Am J Gastroenterology 2006;101:1057-65.

9. Whitehead WE, Levy RL, VanKorff M, et al. The usual medical care for irritable bowel syndrome. Aliment Pharmacol Ther 2004;20:1305-15.

10. Francis CY, Morris J, Whorwell PJ. The irritable bowel severity scoring system: A simple method of monitoring irritable bowel syndrome and its progress. Aliment Pharmacol Ther 1997;11:395-402.

11. Patrick DL, Drossman DA, Frederick HO, et al. Quality of life in persons with irritable bowel syndrome. Development and validation of a new measure. Dig Dis Sci 1998;43:400411. 